\title{
Contribution of Slip and Cleavage in Friction and Wear at (1 0 -1 4) Surface of Magnesite $\left(\mathrm{MgCO}_{3}\right)$ Crystal
}

\author{
Kaori Niki ${ }^{*}$, Mai Kobayashi and Hitoshi Shindo \\ Graduate School of Science and Engineering, Chuo University \\ 1-13-27 Kasuga, Bunkyo-ku, Tokyo 112-8551, Japan \\ *Corresponding author: niki@kc.chuo-u.ac.jp
}

( Manuscript received 1 September 2011; accepted 29 November 2011; published 15 December 2011 )

\begin{abstract}
Frictional force was measured using Bowden-Leben apparatus with a sapphire stylus at directional (1 0 - 14 ) surface of magnesite crystal. Deformation patterns formed during the scratch in \pm [4 2 -1] directions were observed with optical microscopy. With the normal load up to $460 \mathrm{mN}$, no trace of slip or cleavage was observed and the friction coefficient remained at 0.0085 . Frictional asymmetry due to the tilt of carbonate ions was not recognized in this range. At higher normal loads, new components were added to the frictional force, with the appearance of traces of slip, and then, cleavage on $\left\{\begin{array}{llll}1 & 0 & -1 & 4\end{array}\right\}$ faces. In contrast with the case of calcite, traces of the slip remained within the width of the stylus. Traces of the cleavage, on the other hand, reached far outside the wear track, and asymmetry was recognized in the pattern formation. In indentation experiments, the cleavage occurred in four directions, but slip on $\left(\begin{array}{llll}0 & 0 & 0 & 1\end{array}\right)$ was not recognized. In contrast with isostructural but softer calcite crystal, the difficulty in the $\left(\begin{array}{llll}0 & 0 & 0 & 1\end{array}\right)$ slip with magnesite keep the friction coefficient low toward higher normal loads.
\end{abstract}

Keywords: friction coefficient, $\mathrm{MgCO}_{3}$, single crystal, cleavage, frictional asymmetry

\section{Introduction}

In order to understand mechanisms of friction in the atomic scale, friction measurements at single crystal surfaces have been performed using frictional force microscopy $(\mathrm{FFM})^{1,2)}$. Clear-cut results are often obtained with FFM. At $\left(\begin{array}{lll}1 & 0 & 0\end{array}\right)$ and $\left(\begin{array}{lll}1 & 1 & 0\end{array}\right)$ surfaces of alkali halides, for example, lower friction was detected along the scan directions where like ions are aligned ${ }^{3,4)}$. The anisotropy in friction has been expected and studied by many workers. However, using conventional friction apparatus, results were not clear and often betrayed our intuition $^{5-7)}$. Plastic deformation including slip and cleavage probably make situations complex in micrometer- or millimeter-scales.

In wearless conditions in atomic-scale, friction is explained by dissipation of energy stored in the spring systems of stylus-surface in stick-slip actions ${ }^{8-11}$. In larger scales, too, energy dissipation by plastic deformation will be the source of friction. It is, thus, important to know what sorts of plastic deformation work at surface layers of solids, and at what levels of stresses. Where does the friction in larger scales deviate from the friction in the atomic-scale?

Frictional asymmetry has not been widely known in conventional scales. In nano-scale friction, however, clear asymmetry has been observed at single crystal surfaces having tilted $\mathrm{S}=\mathrm{O}$ bonds of sulfate ions ${ }^{12-15)}$, $\mathrm{C}=\mathrm{O}$ bonds of carbonate ions ${ }^{16)}$, or long-chain organic molecules adsorbed ${ }^{17-19)}$. The asymmetry was explained using mechanical models of mass-spring systems ${ }^{15,16)}$.

With ( $\left.\begin{array}{llll}1 & 0 & -1 & 4\end{array}\right)$ surface of calcite crystal $\left(\mathrm{CaCO}_{3}\right.$, Mohs hardness $=3$ ), comparison between frictions in nano-scale and larger scale was possible, since nearly atom-flat surfaces with wide areas are obtained in cleavage. Presence of steps, if any, at the surface does not change the tilt direction of $\mathrm{C}=\mathrm{O}$ bonds. A sketch of the crystal is given in Fig. 1A. Triangular carbonate ions are all tilted to the same direction making an angle of $44.6^{\circ}$ with the cleaved surface.

Frictional asymmetry was clearly detected with FFM with the normal load of $125 \mathrm{nN}$ and with the scan length of $100 \mu \mathrm{m}^{16)}$. Similar asymmetry was observed in friction measurements in millimeter-scale using a Bowden-Leben apparatus with a sapphire stylus of $60^{\circ}$ cone angle, applying the normal load smaller than 49 $\mathrm{mN}^{20)}$. With heavier normal loads, however, reversed frictional asymmetry, - [ [ $\left.\begin{array}{lll}4 & 2 & -1\end{array}\right]>+\left[\begin{array}{lll}4 & 2 & -1\end{array}\right]$, was observed. Triangular deformation structures formed at the surface, only during the scan in the direction of the stronger friction, included dense traces of slip on $c\left(\begin{array}{lll}0 & 0 & 0\end{array}\right.$ 
1) faces and much less frequent cleavage on $r\left\{\begin{array}{llll}1 & 0 & -1 & 4\end{array}\right\}$ side faces. The slip on tilted $c$-faces occurred only on one side of the stylus, and $r$-cleavage followed in the slipped area. The frictional force increased in proportion to the size of the triangles. Along $\pm\left[\begin{array}{lll}0 & 1 & 0\end{array}\right]$ directions, no asymmetry was detected in the friction coefficients and the traces of plastic deformation. We consider that energy dissipation accompanying the $\left(\begin{array}{llll}0 & 0 & 0 & 1\end{array}\right)$ slip gives an additional term of energy dissipation causing increase in the friction coefficient only in one direction.

Since the plastic deformation observed occurred even under fairly small normal loads, we should bear in mind that same things might occur in conventional friction experiments. We need to check if the phenomenon is generally observed with other crystals.
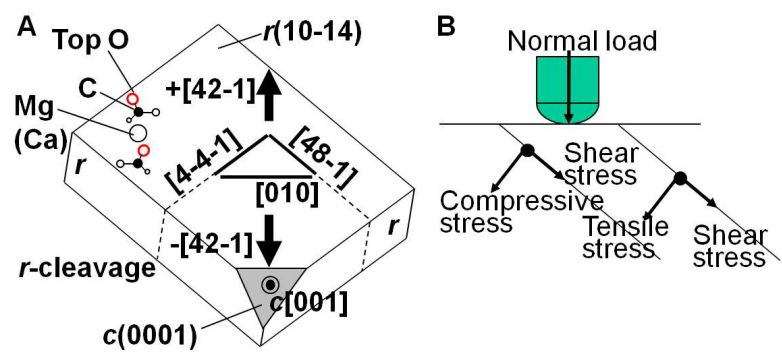

Fig. 1 Sketches showing A: The outer shape of a cleaved $\mathrm{MgCO}_{3}\left(\mathrm{CaCO}_{3}\right)$ crystal, and $\mathbf{B}$ : Mechanical forces working at tilted, potential slip or cleavage surfaces

This time, we have performed similar experiment at

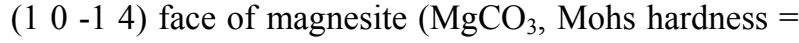
3.5-5), which has a structure similar to calcite but has a slightly shorter ( 0.880 times) separation between the $(0$ $\left.\begin{array}{lll}0 & 0 & 1\end{array}\right)$ ionic layers. In the case of calcite, the $c$-slip dominated the deformation pattern and caused the frictional asymmetry. We can, however, expect a difference with magnesite. By comparing results with the two crystals, we can discuss the mechanism of plastic deformation of materials and its contribution to friction.

\section{Experiments}

Single crystals of calcite and magnesite used were of natural origin. They were cleaved into thin plates by giving light blows on a knife edge placed on the crystal faces. Frictional force was measured using a Bowden-Leben apparatus (HEIDON 18 LFW), using a sapphire stylus with the cone angle of 60 degrees and the curvature radius of $50 \mu \mathrm{m}$. At the cleaved (1 $0-14)$ surfaces, the frictional force was recorded while the stylus was scanned with the speed of $24 \mathrm{~mm} / \mathrm{min}$ and with the scan length of $2 \mathrm{~mm}$ in $+\left[\begin{array}{lll}4 & 2 & -1\end{array}\right]$ or - [ [ $\left.\begin{array}{lll}4 & 2 & -1\end{array}\right]$ direction, applying normal loads of $9.8-980 \mathrm{mN}$. Only a single scan was performed at one place. Wear tracks formed at the surfaces of transparent crystals were observed with HiRox KH-7700 digital microscope after the scans.

Indentation experiments were performed using Shimadzu EZ-S universal testing machine with a stainless steel indenter having a cone angle of $60^{\circ}$. Deformation structures formed were also observed with the optical microscope. Much heavier normal loads were required to cause the plastic deformation in the static experiment, than in the friction experiments.

\section{Results}

Frictional forces measured upon scanning the stylus in $\pm\left[\begin{array}{lll}4 & 2 & -1\end{array}\right]$ direction are plotted against the normal load in Fig. 2. The frictional force increased in proportion to the normal load in the lowest normal load range up to $460 \mathrm{mN}$ (region $\boldsymbol{a}$ ). The frictional coefficient was 0.0085 . Frictional asymmetry was not recognized here. In the normal load range of $500-750 \mathrm{mN}$ (region b), however, a new component was added to the friction in - [ $\left.\begin{array}{lll}4 & 2 & -1\end{array}\right]$ direction and the linear relationship was broken. With even higher normal load (region $c$ ), the frictional force dropped again.

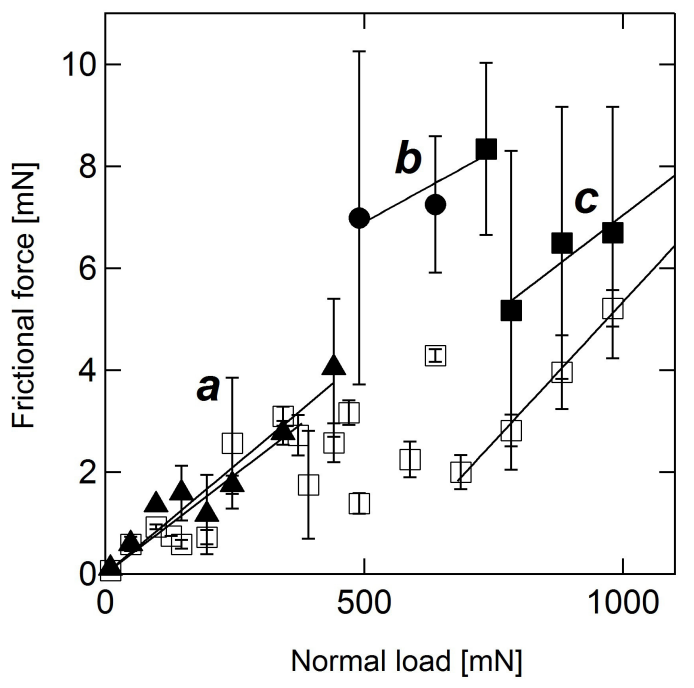

Fig. 2 Frictional force vs. normal load in \pm [4 2 -1] scratch. The $\boldsymbol{\Delta}$ signs show the intrinsic friction in - [4 2 -1] scratch. The $\bullet$ signs in region $\boldsymbol{b}$ and - signs in regions $\boldsymbol{b}$ and $\boldsymbol{c}$ correspond to detection of the $c$-slip and $r$-cleavage, respectively, in - [4 2 -1] scratch. Results for + [ $\left.\begin{array}{lll}4 & 2 & -1\end{array}\right]$ scratch are indicated by the $\square$ signs.

During the scratch in $+\left[\begin{array}{lll}4 & 2 & -1\end{array}\right]$ direction, frictional force was smaller in the regions $\boldsymbol{b}$ and $\boldsymbol{c}$. There is some asymmetry in friction in the higher normal load regions.

Traces formed by the scratch at the magnesite surface were observed with the optical microscope, then. In the normal load range of $9.8-441 \mathrm{mN}$ (region $\boldsymbol{a}$ ), no wear track was observed. The image in Fig. 3A was observed after - [ $\left.\begin{array}{lll}4 & 2 & -1\end{array}\right]$ scratch with the normal load of $490 \mathrm{mN}$ (region $\boldsymbol{b}$ ). Traces along the [lll $\left.\begin{array}{lll}0 & 1 & 0\end{array}\right]$ direction 
were detected. Since the traces remained within the wear track, they must have been formed by direct contact with the stylus. The jump in the frictional force in region $\boldsymbol{b}$ in Fig. 2 most probably corresponds to the formation of the traces. Let us consider the origin of the traces.

Various slip surfaces were reported in literature for calcite $^{21,22)}$. Among them, the slip on $c\left(\begin{array}{llll}0 & 0 & 0 & 1\end{array}\right)$ face was the one which gave lots of traces in $\left[\begin{array}{lll}0 & 1 & 0\end{array}\right]$ direction in

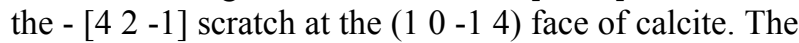
traces were formed under the normal load as small as 49 $\mathrm{mN}^{20)}$, and extended well out of the wear track. No trace, however, was formed in $+\left[\begin{array}{lll}4 & 2 & -1\end{array}\right]$ scratch. It will be reasonable to assume the same slip system with magnesite.

With the harder magnesite, however, the traces in $[0$ $10]$ direction were formed under much heavier normal load and only with direct contact with the stylus. The traces were formed both in $+\left[\begin{array}{lll}4 & 2 & -1\end{array}\right]$ and - [ [ $\left.\begin{array}{lll}4 & 2 & -1\end{array}\right]$ scratch as shown in Fig. 3.

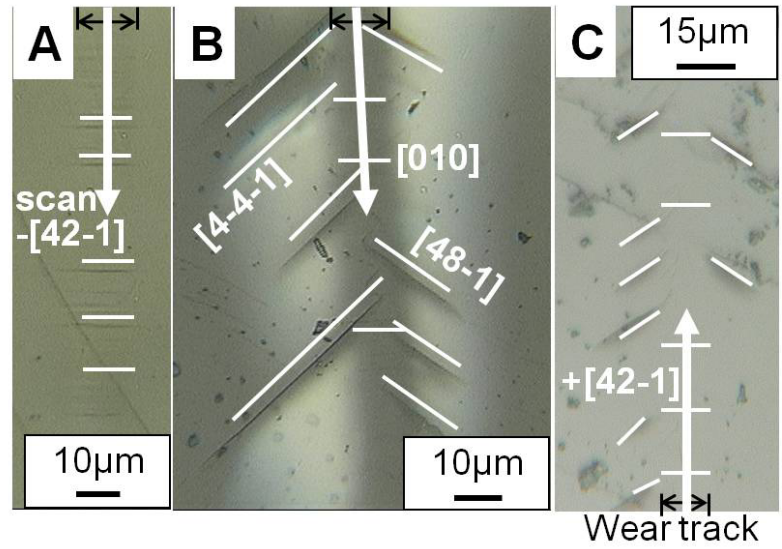

Fig. 3 Optical microscope images of $\mathrm{MgCO}_{3}\left(\begin{array}{llll}1 & 0 & -1 & 4\end{array}\right)$ face after scratching with normal loads of $\mathbf{A}$. $490 \mathrm{mN}$ and B.980mN in - [4 2 -1] direction, and C.980 $\mathrm{mN}$ in $+\left[\begin{array}{ll}4 & 2 \\ -1\end{array}\right]$ direction. Traces of $\left[\begin{array}{lll}0 & 1 & 0\end{array}\right]$ direction and cracks in the [4 -4 -1] and [4 8 -1] directions are indicated by white line segments drawn nearby.

The image in Fig. 3B was observed after the scratch in - [ $\left.\begin{array}{lll}4 & 2 & -1\end{array}\right]$ direction with the normal load of $980 \mathrm{mN}$ (region $c$ ). In this range of normal load, the traces in $[0$ $10]$ direction became less obvious and cracks in [4 -4 -1] and [4 8 -1] directions, parallel to the edges of the crystal, were clearly detected. These cracks were formed by cleavage on $r\left\{\begin{array}{llll}1 & 0 & -1 & 4\end{array}\right\}$ faces parallel to the side faces of the crystal shown in Fig. 1A. They extended far outside the actual wear track. The lengths of the cleavage lines increased with the normal load.

In the case of the calcite surface, dense slip lines in $\left[\begin{array}{lll}0 & 1 & 0\end{array}\right]$ direction and the cleavage lines together made triangular deformation patterns in the - [ $\left.\begin{array}{lll}4 & 2 & -1\end{array}\right]$ scratch, but not in the $+\left[\begin{array}{ll}4 & 2\end{array}-1\right]$ scratch. In the case of magnesite, however, the slip and cleavage occurred independently. In addition, similar cracks were formed, as shown in Fig.

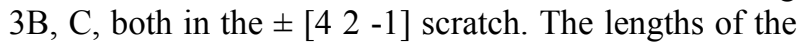
cleavage lines, however, are shorter in the $+\left[\begin{array}{lll}4 & 2 & -1\end{array}\right]$ scratch.

In order to compare bulk deformation properties of the two crystals, indentation experiments were performed by applying much heavier normal load with a stainless steel indenter. The optical microscope image shown in Fig. 4A was observed at the (1 $0-14)$ surfaces of calcite $\left(\mathrm{CaCO}_{3}\right)$ after indentation with the normal loads of $40 \mathrm{~N}$. A triangular deformation pattern was formed only on one side of the indenter. The cleavage lines denoted $\mathbf{a}$ and $\mathbf{b}$ in [4 -4 -1] and [4 8 -1] directions, make two shorter sides of the triangle. Quite many slip lines $\mathbf{c}$ in $\left[\begin{array}{lll}0 & 1 & 0\end{array}\right]$ direction make the bases. The slip surface giving the lines $\mathbf{c}$ was determined to be the $c\left(\begin{array}{ll}0 & 0\end{array}\right.$ 0 1) surface by measuring the tilt angle from the indented face with microscope images observed in horizontal direction ${ }^{20)}$. Similar patterns were formed in the friction experiment along the - [4 2 -1] direction.

The result for $\mathrm{MgCO}_{3}$ surface with the normal load of $40 \mathrm{~N}$ is shown in Fig. 4B. Here, only cleavage cracks, but no slip lines in $\left[\begin{array}{lll}0 & 1 & 0\end{array}\right]$ direction, were observed. The cleavage cracks $\mathbf{a}$ and $\mathbf{b}$ were formed even with the normal load as small as $1 \mathrm{~N}$. The cracks a' and 'b' were formed with the load $>10 \mathrm{~N}$.
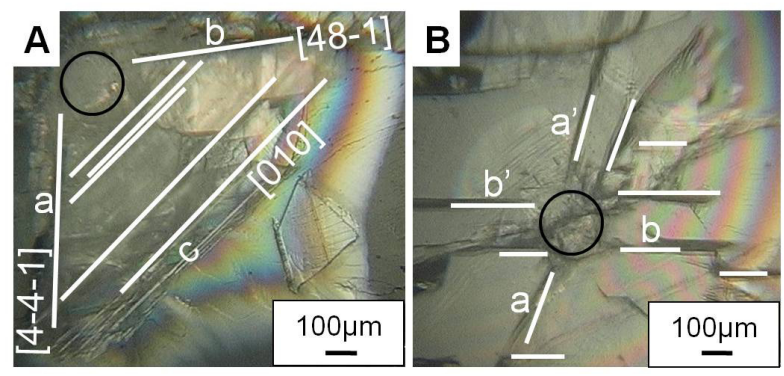

Fig. 4 Optical microscope images of (1 $0-14)$ faces of, $\mathbf{A} \cdot \mathrm{CaCO}_{3}$ and $\mathbf{B} \cdot \mathrm{MgCO}_{3}$, after indentation experiment with normal loads of $40 \mathrm{~N}$. Traces of $r\left\{\begin{array}{llll}1 & 0 & -1 & 4\end{array}\right\}$ cleavages are indicated by $\mathbf{a}, \mathbf{b}$, a' and b'. The traces of $c\left(\begin{array}{llll}0 & 0 & 0 & 1\end{array}\right)$ slips are indicated by c. Compressed points are indicated by circles.

\section{Discussion}

The main difference between the two rhombohedral crystal structures of calcite and magnesite lies in the interlayer separation between the $c\left(\begin{array}{llll}0 & 0 & 0 & 1\end{array}\right)$ faces. The $c$-face is an electrically polar face consisted of either cations or triangular carbonate ions lying flat. The slip occurs between the cation layer and the anion layer. In the friction experiment at the calcite( $10-14)$ surface, the $c$-slip occurred easily prior to $r$-cleavage, giving a characteristic triangular deformation patterns like the one shown in Fig. $4 \mathrm{~A}^{20}$ ). 
A mechanical model of a slip at tilted interfaces is shown in Fig. 1B. Depending on the positions of the potential slip surfaces relative to the compressed point, tensile stress helping the slip, or compressive stress preventing the slip, works in addition to the shear stress. The slip, then, occurs preferably on one side of the crystal face. This explains why the $c$-slip with calcite occurs only on one side of the stylus as shown in Fig. 4A. The cleavage on $r$-faces follows and forms the boundaries $\mathbf{a}$ and $\mathbf{b}$. In the friction experiment, the pattern formation was observed only in - [ [ $\left.\begin{array}{lll}4 & 2 & -1\end{array}\right]$ scratch with marked increase in the friction coefficient. The tilted slip surface caused the frictional asymmetry with calcite.

Since the tilt angles of $c$-face from $r$-face for the two crystals are close to each other $\left(43.8^{\circ}\right.$ for magnesite and $44.6^{\circ}$ for calcite), we can expect the $c$-slip in friction experiments with magnesite, too. However, shorter separation between the cationic and anionic layers may prohibit the slip. In reality, $c$-slip was not observed in Fig. 4B in the indentation experiment with magnesite. Cleavage occurs before the $c$-slip is activated with this crystal.

In Fig. 3A, traces in $\left[\begin{array}{lll}0 & 1 & 0\end{array}\right]$ direction was observed in the friction experiment. Since the traces remain within the width of the stylus, they were formed by very strong interaction with the stylus. Since surface deformation is pronounced, especially in front of the moving stylus, slips, not observed in the indentation experiment, may occur in the friction experiment with much smaller normal load.

The jump in the frictional force in region $\boldsymbol{b}$ of Fig. 2 is most probably caused by the energy dissipation accompanying the slip within the wear track. Although the direction of the slip surface is not confirmed with magnesite yet, it will be reasonable to assume the $c$-slip detected with the isostructural calcite. The difference with calcite is that the traces were formed with

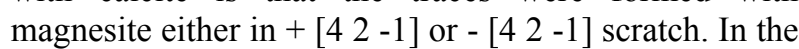
case of calcite, the slip occurred only in - [ [ $\left.\begin{array}{lll}4 & 2 & -1\end{array}\right]$ scratch, on the front side of the stylus. If the same $c$-slip occurred with magnesite in $+\left[\begin{array}{lll}4 & 2 & -1\end{array}\right]$ scratch, it should have occurred on the rear side of the stylus.

Assuming the $c$-slip with magnesite, the frictional asymmetry observed in region $\boldsymbol{b}$ of Fig. 2 is explained as follows. In the - [ [ $\left.\begin{array}{lll}4 & 2 & -1\end{array}\right]$ scratch, the slip makes an upward step to the front side of the moving stylus. Extra energy is required for the stylus to climb up the step and travel further. That is why the frictional force jumped in region $\boldsymbol{b}$. In the $+\left[\begin{array}{lll}4 & 2 & -1\end{array}\right]$ scratch on the other hand, the $c$-slip makes steps only on the rear side of the moving stylus. No extra energy is required for the stylus to travel further. That is why the friction is low in region $\boldsymbol{b}$ in this direction.

The cleavage patterns in Fig. 3B, C show asymmetry in the scan direction. Clearly much longer cleavage cracks were observed in Fig. 3B in the - [ $\left.\begin{array}{lll}4 & 2 & -1\end{array}\right]$ scan. Only short cracks just outside the wear track were formed in Fig. 3C in the $+\left[\begin{array}{lll}4 & 2 & -1\end{array}\right]$ scan.

In the case of calcite, the cleavage lines were observed only in areas where the $c$-slip split the surface into thin strips beforehand ${ }^{20}$.The $r$-cleavage with magnesite, on the other hand, occurs independently. But why do the cleavage lines run in the same direction in the opposite scans? As was seen in Fig. $4 \mathrm{~B}$, cleavage is possible in 4 directions.

The equivalent $r$-faces intersect with each other making angles of $72.6^{\circ}$ for magnesite and $74.9^{\circ}$ for calcite. The asymmetry in the $r$-cleavage with magnesite can also be explained using the mechanical model of tilted interface in Fig. 1B. Since cleavage occurs mainly by the tensile stress between the two sides of the interface, it occurs preferably on one side of the compressed point.

Considering the tilt directions of the $r$-side faces in Fig. 1A, we can expect the pattern of cleavage. Here we assume that cleavage start at defects randomly distributed in the crystal. If the stylus is moving in - [4 2 -1] direction, the cleavage will start at a defect located toward the front side of the moving stylus. The cleavage will proceed while the stylus approaches and finally bump on the cleavage step. The longer cleavage cracks in Fig. 3B and larger energy dissipation are explained by this mechanism. Extra energy is required for the stylus to climb the step.

In the opposite scan in $+\left[\begin{array}{ll}4 & 2\end{array}-1\right]$ direction, however, the cleavage will occur only on the rear side of the stylus. Since the stylus is receding from the cleavage line, the cleavage proceeds only for a short distance as was seen in Fig. 3C. In addition, the stylus need not climb up the cleavage steps formed on the rear side. It is reasonable that the friction is weaker in this direction in region $c$ in Fig. 2.

But how can we explain the drop from region $\boldsymbol{b}$ to region $c$ in the frictional force in the - [4 2 -1] direction? We consider that the lower friction in region $c$ is related to the formation of the long cleavage cracks shown in Fig. 3B. While the cleavage proceeds in front of the moving stylus, the part of surface in contact with the stylus moves downward during the scratch. The net normal load working between the stylus and the sinking surface will be much smaller than the normal load applied by the friction apparatus. The frictional force measured will be smaller than that measured on the static surface. Of course, stronger force will be required to climb up the cleavage steps. Most of the time, however, the stylus enjoys lower friction.

The same explanation will be possible for the low friction in $+\left[\begin{array}{lll}4 & 2 & -1\end{array}\right]$ direction in region $\boldsymbol{b}$ of Fig. 2 . The slipped part of the surface moving downward will give lower friction compared to the static surface.

Finally, we want to compare the friction coefficients of the two crystals, softer calcite and harder magnesite. At the calcite surface, friction coefficients of 0.010 and 0.016 were obtained with - [4 2-1] and + [4 2-1] scans, respectively, with normal load $<49 \mathrm{mN}$. The observed 
asymmetry was similar to the results of FFM in micro-meter-scale. At higher normal loads, with the start of the $c$-slip, the slope of the frictional force vs. normal load curve in - [4 2 -1] increased 6-times to 0.060, while the value in $+\left[\begin{array}{lll}4 & 2 & -1\end{array}\right]$ direction remained the same. Energy dissipation accompanying the slip becomes the main cause of friction at the calcite face. The slip density increases in proportion to the normal load.

When a crystalline solid has a slip system, it may give considerable effects upon friction. The scan of a stylus gives much stronger stress than static compression.

In the case of magnesite, the $c$-slip is not activated without applying quite heavy normal load. The frictional coefficient of 0.0085 in \pm [4 2 -1], not so small a value compared to calcite, remains the same up to the normal load of $460 \mathrm{mN}$. Somehow the frictional asymmetry was not detected.

With the start of slip within the wear track and $r$-cleavage outside, jumps up were observed in the frictional force vs. normal load curve. The jump in region $\boldsymbol{b}$ of Fig. 2 is caused by the energy dissipation accompanying the slip. However, no increase was recognized with the slope. The occasional slip and cleavage cannot be the main component of friction.

By comparing friction and deformation properties of two resembling crystals, we found when and how the bulk structures start to affect the friction. It is amazing that a small difference in structure parameters makes a big difference in friction and wear properties.

\section{Summary}

At $\mathrm{MgCO}_{3}\left(\begin{array}{llll}1 & 0 & -1 & 4\end{array}\right)$ surface, the frictional coefficient measured both in $+\left[\begin{array}{lll}4 & 2 & -1\end{array}\right]$ and - [ $\left[\begin{array}{lll}4 & 2 & -1\end{array}\right]$ directions remained low at 0.0085 with the normal loads $<460 \mathrm{mN}$. No wear track was observed in $\pm\left[\begin{array}{lll}4 & 2 & -1\end{array}\right]$ directions and no frictional asymmetry was recognized.

In the range of $500-750 \mathrm{mN}$, an increase in the friction force was detected only in the - [ [ $\left.\begin{array}{lll}4 & 2 & -1\end{array}\right]$ direction due to the energy dissipation accompanying the slip in $\left[\begin{array}{lll}0 & 1 & 0\end{array}\right]$ direction. Assuming $c\left(\begin{array}{llll}0 & 0 & 0 & 1\end{array}\right)$ as the slip surface, the frictional asymmetry was explained by a mechanical model of slip on tilted interfaces.

With the normal load $>750 \mathrm{mN}$, traces of cleavage extending outside the wear track were formed. In contrast with the case of calcite, the cleavage on $r\{10$ -14 faces occurred without prior slip on the $c$-faces. Asymmetry was detected in the cleavage pattern.

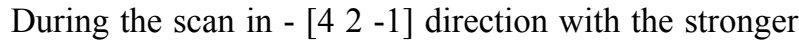
friction, much longer traces of cleavage were formed indicating more energy consumption.

Slight differences between the structures of similar carbonate crystals give marked differences in mechanical properties including friction. Tilted slip and cleavage faces in crystals may give asymmetry in friction.

\section{Acknowledgements}

This work was supported by JSPS KAKENHI (20510097, 20225002), Nitiki Saneyoshi Foundation, and The Institute of Science and Engineering, Chuo University.

\section{References}

[1] Wiesendanger, R., "Scanning Probe Microscopy and Spectroscopy," Cambridge University Press, Cambridge, 1994, 231-235.

[2] Meyer, E., Lüthi, R., Howald, L. and Güntherodt, H. J., "Friction Force Microscopy," in: Güntherodt, H. J., Anselmetti, D. and Meyer, E., editors, "Forces in Scanning Probe Methods," Kluwer, Dordrecht, 1995, 285-306.

[3] Namai, Y. and Shindo, H., "Frictional Force Microscopic Anisotropy on (llll $\left.0 \begin{array}{ll}0 & 1\end{array}\right)$ Surfaces of Alkali Halides and MgO,” Jpn. J. Appl. Phys. B, 39, 2000, 4497-4500.

[4] Karino, W. and Shindo, H., "Frictional Force Microscopic Detection of Anisotropy at $\mathrm{NaCl}(10$ 0), ( $\left.\begin{array}{lll}1 & 1 & 0\end{array}\right)$ and ( $\left(\begin{array}{lll}1 & 1 & 1\end{array}\right)$ Surfaces," Tribology International, 40, 2007, 1568-1573.

[5] Steijn, R. P., "Friction and Wear of Single Crystals," Wear, 7, 1964, 48-66.

[6] Bowden, F. P. and Brookes, C. A., "Frictional Anisotropy in Nonmetallic Crystals," Proc. Roy. Soc. London A, 295, 1966, 244-258.

[7] Kobayashi, S., Okui, T. and Miura, S., "Microscopic Deformations in KC1 Single Crystals by Indentation and Scratching with Steel Ball and Pyramidal Indenters," Wear, 162-164, 1993, 92-101.

[8] McClelland, G. M., "Adhesion Friction at Weakly Interacting Interfaces," in: Grunze, M. and Kreuzer, H. J., editors, "Adhesion and Friction," Springer, Berlin, 1990, 1-16.

[9] McClelland, G. M. and Glosli, G. M., "Friction at the Atomic Scale," in: Singer, I. L. and Pollock, H. M., editors, "Fundamentals of Friction: Macroscopic and Microscopic Processes," Kluwer, Dordrecht, 1992, 405-425.

[10] Tománek, D., Zhong, W. and Thomas, H., "Calculation of an Atomically Modulated Friction Force in Atomic-Force Microscopy," Europhysics Letters, 15, 8, 1991, 887-892.

[11] Tománek, D., "Theory of Atomic-Scale Friction," in: Wiesendanger, R. and Güntherodt, H. J., editors, "Scanning Tunneling Microscopy III," Springer, Berlin, 1993, 269-292.

[12] Bluhm, H., Schwarz, U. D., Meyer, K. P. and Wiesendanger, R., "Anisotropy of Sliding Friction on the Triglycine Sulfate (0 10 0) Surface," Appl. Phys. A, 61, 1995, 525-533.

[13] Bluhm, H., Schwarz, U. D. and Wiesendanger, R., "Origin of the Ferroelectric Domain Contrast 
Observed in Lateral Force Microscopy," Phys. Rev. B, 57, 1998, 161-169.

[14] Shindo, H., Shitagami, K., Sugai, T. and Kondo, S., "Evidence of the Contributions of Molecular Orientations on the Surface Force Friction of Alkaline Earth Sulfate Crystals," Phys. Chem. Chem. Phys., 1, 1999, 1597-1600.

[15] Shindo, H., Shitagami, K., Sugai, T. and Kondo, S., "Detection Mechanism of S-O Tilt Directions on $\mathrm{CaSO}_{4}\left(\begin{array}{lll}1 & 0 & 0\end{array}\right)$ with Frictional Force Microscopy," Jpn. J. Appl. Phys. B, 39, 2000, 4501-4503.

[16] Kwak, M. and Shindo, H., "Frictional Force Microscopic Detection of Frictional Asymmetry and Anisotropy at (1 $0-1$ 4) Surface of Calcite," Phys. Chem. Chem. Phys., 6, 2004, 129-133.

[17] Overney, R. M., Takano, H., Fujihira, M., Paulus, W. and Ringsdorf, H., "Anisotropy in Friction and Molecular Stick-Slip Motion," Physical Review Letters, 72, 1994, 3546-3549.

[18] Gourdon, D., Burnham, N. A., Kulik, A., Dupas, E., Oulevey, F., Gremaud, G., Stamou, D., Liley, M., Dienes, Z., Vogel, H. and Duschl, C., "The
Dependence of Friction Anisotropies on the Molecular Organization of LB Films as Observed by AFM," Tribology Letters, 3, 1997, 317-324.

[19] Liley, M., Gourdon, D., Stamou, D., Meseth, U., Fischer, T. M., Lautz, C., Stahlberg, H., Vogel, H., Burnham, N. A. and Duschl, C., "Friction Anisotropy and Asymmetry of a Compliant Monolayer Induced by a Small Molecular Tilt," Science, 280, 1998, 273-275.

[20] Niki, K., Kobayashi, M. and Shindo, H., "Frictional Asymmetry and Wear Pattern Formation by Slip and Cleavage Detected at Directional $r\left\{\begin{array}{llll}1 & 0 & -1 & 4\end{array}\right\}$ Face of Calcite $\left(\mathrm{CaCO}_{3}\right)$," submitted.

[21] De Bresser, J. H. P. and Spiers, C. J., "Strength Characteristics of the $r, f$, and $c$ Slip Systems in Calcite," Tectonophys. 272, 1997, 1-23.

[22] Barber, D. J., Wenk, H. R., Gomez-Barreiro, J., Rybacki, E. and Dresen, G., "Basal Slip and Texture Development in Calcite: New Results from Torsion Experiments," Phys. Chem. Minerals, 34, 2007, 73-84. 


\section{ERRATA}

Volume and issue: Vol.6, No.7 (2011)

Page: pp.317-322

Title: Contribution of Slip and Cleavage in Friction and Wear at (10-14) Surface of Magnesite $\left(\mathrm{MgCO}_{3}\right)$ Crystal Author(s): Kaori Niki, Mai Kobayashi and Hitoshi Shindo

\begin{tabular}{|ll|l|l|}
\hline & Appearing & Corrected \\
\hline p.318 & Ordinate of Fig. 2 & Frictional force $[\mathrm{mN}]$ & Frictional force [gf $(=9.81 \mathrm{mN})]$ \\
\hline p.318 & right column, line16 & 0.0085 & 0.085 \\
\hline $\begin{array}{l}\text { p.320 } \\
\begin{array}{l}\text { right column, } \\
\text { lines 2-3 from bottom }\end{array}\end{array}$ 0.010 and 0.016 & 0.10 and 0.16 \\
\hline p.321 & left column, line 4 & 0.060 & 0.60 \\
\hline & left column, line 15 & 0.0085 & 0.085 \\
\hline
\end{tabular}

Volume and issue: Vol.7, No.1 (2012)

Page: pp.8-12

Title: Frictional Asymmetry and Wear Pattern Formation by Slip and Cleavage Detected at Directional $r$ \{10-14\} Face of Calcite $\left(\mathrm{CaCO}_{3}\right)$

Author(s): Kaori Niki, Mai Kobayashi and Hitoshi Shindo

\begin{tabular}{|l|l|l|}
\hline & Appearing & Corrected \\
\hline p.9 $\quad$ Ordinate of Fig.2 & Frictional force $[\mathrm{mN}]$ & Frictional force [gf $(=9.81 \mathrm{mN})]$ \\
\hline
\end{tabular}

Errata published; 30, April, 2014 